\title{
PUBLIC ON LINE DELIBERATION SYSTEM (PODS)
}

\author{
Vassilis Triantafyllou, Vassilios Tsakanikas, George Asimakopoulos and John Christodoulopoulos \\ Computer Informatics and Engineering Department, Technological Educational Institute of Western Greece \\ GR-30200, Antirrio, Greece
}

\begin{abstract}
Within this manuscript, we present a set of fundamental and advanced issues regarding a public on line deliberation system, which is based on Public Deliberation basic principles. We present the architecture of a system named PODS (Public On-Line Deliberation System). PODS allows decision makers (also called authority) or citizens to propose a theme for deliberation and results in presenting a winning proposal about an issue. The system supports the upload of a deliberation theme, proposal setting and an iterative process which leads to a winning proposal. Citizens can be categorized by various criteria and provide their opinions via comments or relevant documentation and vote. The whole process is transparent to public through rating and publication and take into account political analytics.
\end{abstract}

\section{KEYWORDS}

Outvoted Proposal Political Analytics, Public Deliberation, Voting

\section{INTRODUCTION}

The development of e-government is a major technological achievement allowing citizens to participate equally in the political process. E-government as a part of e-democracy encompasses social, economic and cultural conditions that enable the free and equal practice of political self-determination. In practice, e-democracy is based on two pillars to be successful - one is e-participation and the other is e-voting- according the "Characterizing E-participation in Policy-Making" (Macintosh, 2004) and has implementation in different countries.

ICT can be a powerful tool to increase the quality of public policy, citizens understanding of public policy issues and their participation in government policy making. In the report from the Government Online International Network, it is presented that governments around the globe are actively promoting online consultations in order to strengthen democracy and development (Poland, 2001). To achieve this, the governments have to:

- Ensure access to and accessibility of national government information.

- Conduct pilot projects in the field of electronic public consultation / deliberation.

- Coordinate the pilot projects, compiling the resulting experiences and developing a certain standard for online public consultation / deliberation.

- Formally adjust the policy process: making changes in institutions and legislation.

A definition of e-participation refers to e-participation as "the use of information and communication technologies to broaden and deepen political participation by enabling citizens to be connected with one another and with their elected representatives" (Macintosh A. , 2006). The form of e-participation is the distinction between government initiated / top - down approaches and citizens initiated / bottom - up approaches.

According to Macintosh (Macintosh A. , 2004), the main objectives of e-participation are: "(a) reach a wider audience to enable broader participation, (b) support participation through a range of technologies to cater for the diverse technical and communicative skills of citizens, (c) provide relevant information in a format that is both more accessible and more understandable to the target audience to enable more informed contributions and (d) engage with a wider audience to enable deeper contributions and support deliberative debate. 
In section 2 we present and describe the e-consultation / deliberation process and our system, while in section 3 we present state-of-the-art online deliberation systems. In section 4 we talk about the Basics Admission of our system while in section $\mathrm{V}$ we see the architecture of our system. Finally, we see conclusions and future work.

\section{PUBLIC ON LINE DELIBERATION}

Before presenting the fundamental principles of online deliberation, one should state what is not online deliberation, in order to assist the reader to clear out the following concepts. Thus, online deliberation should be dissociated from a simple voting procedure or a questionnaire session. While voting collects the opinions of the citizens on specific predefined questions, a deliberation system provides a workflow for formulating and supporting a proposal for a theme. For example, while a voting system would be suitable for collecting citizens' opinions on the question: "should the new medical center of Patra be build next to the city hall?", a deliberation system would publish and support a theme like "Where should the new medical center of Patra be build?". Thus, while the output of a voting system would be a set of percentages describing citizens' opinions, a deliberation system would provide a proposal (winning) regarding a theme. Likely, while a questionnaire extends the possibilities of a voting session, the output is far more restricted than a deliberation process.

Public Deliberation is the dynamic process of dialogue between individuals or groups, based on a genuine exchange of views, and normally with the objective of influencing decisions, policies or programmers of action (Institute C. , 2010). So e-deliberation is an online deliberation process using the Internet to obtain from the public their opinion on one or more specific topics, allow and enhance the discussion between citizens and formulate a set of widely excepted proposals. E-deliberation poses the use of electronic computing and communication technologies in the deliberation processes and is complimentary to existing practices. E-deliberation can be an effective tool in encouraging participation and gathering responses to consultation documents and social policy issues as part of a broader range of methodologies (Institute C. , 2010). Deliberation has been introduced in the Greek legislation as a tool for local governments since 2009 as a tool for increasing citizens' participation.

\subsection{Basic Principles for a Successful E-Deliberation}

Deliberation is an approach to decision-making that allows participants to take under consideration relevant information, discuss the issues and options and develop a common approach resulting to a favorable decision.

INVOLVE (Council, 2008), a public participation charity, states that deliberative public engagement can be used:

- across all levels of government, local, regional, national and international;

- $\quad$ across all types of services, delivered by public, private or voluntary sectors;

- across the spectrum of participation, to inform, consult, involve or empower people

- alongside other forms of participation such as, opinion polls, written consultations, community development, campaigning or lobbying;

- $\quad$ at any point in the policy cycle:

$\circ$ when an issue is initially identified as being of concern (policy determination or agenda-setting);

- when the process for tackling the issue and potential outcomes are set (policy direction);

$\circ$ in planning the key elements of the desired outcomes and how to achieve them (policy design);

$\bigcirc$ or during implementation, monitoring and review (policy delivery).

There are 9 basic principles which determine a successful e-deliberation (Council, 2008):

- The process makes a difference to participants, to decision making policy, to policy makers through identifying a clear task or purpose and presenting the potential consequences / results.

- The process is transparent. It is essential to ensure that the procedure is accessible to as many as possibly interested individuals engaging a range of people and information sources - including information, evidence and views from people with different perspectives, backgrounds and 
interests and that minority or disadvantaged groups are not excluded, or that discussions are not dominated by any particular faction. All involved parties are aware of how policy and decision makers will use their contribution.

- The process has integrity. The process must give participants sufficient time and space to enable them to gain new information and to discuss in depth the implications and width of their new knowledge in terms of their existing attitudes, values and experience. All the interested parties ought to trust each other's motives and the specific procedure, even if necessarily a desirable content cannot be reached.

- The process is tailored to circumstances. It is crucial that the purpose and objectives (why and how), the intended outcomes (what are the expected results), the people that should be involved (specialists, stakeholders) and different needs (location) and aspirations (active citizens, politicians) and the context into which the process will fit in.

- The process involves the right number and types of people. All citizens (participants) of different ages, gender, social class, ethnic groups, geographical location, expertise, should be involved ensuring demographic representation.

- The process treats participants with respect. All participants must feel that their contribution and needs are clearly valued. Relevant policy and decision-makers must take part directly in the process and share a clearly state commitment of taking the process seriously. The process must provide a safe atmosphere in which they can express their views freely and a friendly and informal environment.

- The process values participants' discussions. The main focus should be that the process follows a logical path through learning and discussion, allows feedback and summing up so that expect results are clearly interpreted by decision makers.

- The process is reviewed and evaluated to improve practice. Effective evaluation (as early as possible in the process), synthesis and achievement of measurable results are essential.

- Participants are kept informed. Participants are informed about intermediate results and final decisions.

\section{CURRENT STATUS OF ONLINE DELIBERATION SYSTEMS}

A number of online deliberation systems have been deployed worldwide during the last decade, a fact which reveals that need for creating more easy-to-use intelligent systems for direct democracy procedures.

A system which falls in the category of online deliberation systems is liquidfeedback ${ }^{1}$, which provides users the possibility of either creating or supporting initiatives. While liquidfeedback provides a complete set of tools, like propose initiative, comment initiative and support initiative, it does not offer the possibility of setting up criteria for an initiative, making it unappropriated for large scale deliberations. The same disadvantage appears on debatehub ${ }^{2}$, which is an open, online, collaborative tool to support collective ideation, deliberation and democratic decision making. Similar tools and formats appear on several other systems, like Decide Madrid $^{3}$, GovTogetherBC 4 , Irekia ${ }^{5}$ and Finnish citizens' initiative ${ }^{6}$. Finally, MOODS (Verdiesen, 2016) incorporates tools for managing large-scale deliberations and proposes some really interesting aspects towards this direction. The aforementioned systems share the same drawbacks. They don't provide the necessary tools for supporting large-scale deliberations and they don't offer any intelligent tools for post-processing the results and formulating the winning proposal/initiative. PODS, as presented in the following sections, attempts to overcome these drawbacks by integrating innovative workflows and intelligent modules to an online deliberation system, offering a set of tools and reasoning mechanisms for producing the final output, based on a multi-criteria analysis of data coming from user profile, social networks or geolocation.

\footnotetext{
${ }^{1}$ https://liquidfeedback.org/

${ }^{2}$ https://debatehub.net

${ }^{3}$ https://decide.madrid.es/

${ }^{4}$ https://engage.gov.bc.ca/govtogetherbc/

${ }^{5} \mathrm{https} / / / \mathrm{www}$.irekia.euskadi.eus

${ }^{6}$ https://vrk.fi/en/finnish-citizens-initiative
} 


\section{PODS - AN INTERGRATED APPROACH FOR ON LINE DELIBERATION}

The formulation of an integrated approach to online deliberation led to the design and implementation of the PODS (Public On-line Deliberation System). The system is based on the principles presented in the previous section and on four, functional modules, which contribute to the proper and effective functioning of the PODS model.

- Information Base. The Information Base is the primary functional module where all data are stored and retrieved from through the deliberation process. This includes personal information of the participants (citizens), such as name, address, gender, age, identity, expertise, social profile and other parameters that during the process determine the analytics used to surface the most prevalent proposal/suggestion. This allows citizens to be categorized by various criteria.

- Discussion forum. The deliberation uses a type of "electronic forum" in which participants can express their opinions through the relevant website and engage in the dialogue and exchange of opinions between them. Basic rules of civilized dialogue are applied to ensure equal opportunities of participation, encourage involvement of citizens who are reluctant to express themselves, and limit those who tend to monopolize the debate. These rules are known ex - ante and compliance to these rules is the responsibility of an independent adjudicator, the debate moderator.

- Evaluation procedure. Evaluation of the proposals is based on a composite procedure that highlights "winning proposals". The procedure allows each participants to grade each proposal based on a set of decision criteria (Drakopoulos, Triantafyllou, \& Christodoulos, 2018) such as positive, negative, neutral votes, social factors, (citizens' digital reputation, participation frequency and innovation, geolocation etc.). After a number of consequent rounds, where proposals with low acceptance are discarded or proposals with similar context can be merged a set of "winning proposals" are surfaced.

- Deliberation Authority. The PODS system is monitored by a moderation body which is responsible to set up the criteria and coefficients to be used by the evaluation procedure to facilitate the screening process towards awarding the "winning proposals". The criteria and coefficients introduce the "political view" of local authorities.

All subjects under deliberation are accompanied by a basic set of documentation that allows all participants to understand the significance, restrictions and impact of the subject. The policy makers take into account the deliberation results, other factors like time and budget constraints, impact on other policies etc.

As depicted in Figure 1, the PODS system involves 3 type of users:

- Deliberation authorities: These include all authorities that introduce a deliberation. The PODS system is mainly addressed to authorities (local council, public bodies or groups, management executives etc.), that need to deliberate on a concept / problem / issue allowing the participants / citizens to express their opinions and evaluate possible proposals. The authority can issue an open call about issues that are significant to the public and it is expected that the deliberation process will identify solutions that can sum up the majority of the participants. All issues proposed by non authorized groups are under the acceptance of the deliberation authority.

- Proposers: Any participant / citizen can present a proposal / opinion about the theme introduced for deliberation. The proposers presents their proposal including any documentation needed to support their thesis and are responsible to work with other proposers in order to merge their proposition as a result of the evaluation procedure.

- Citizens / Participants: The participants is a closed set of individuals that participate in the deliberation process based on their identity (citizens, interested people, members of a specific group, etc.). Each participant logs in using a username, password, and the user inserts all data at relevant fields that correspond to relevant criteria and factors the authority body believes should be a part of the evaluation procedure. The final decisions are biased by the factors and criteria agreed to be considered as part of a specific deliberation process.

In terms of the technological aspects, PODS utilizes REST APIs for integrating with external user repositories, in order to provide secure user authentication. For data storage, PODS follows a hybrid approach, integrating a no-SQL database (mongo- $\mathrm{db}$ ) for storing material related to the proposals and the users' comments and a relational $\mathrm{db}$ for storing the system settings, the produced analytics and other information related to the deployed deliberation setups. 


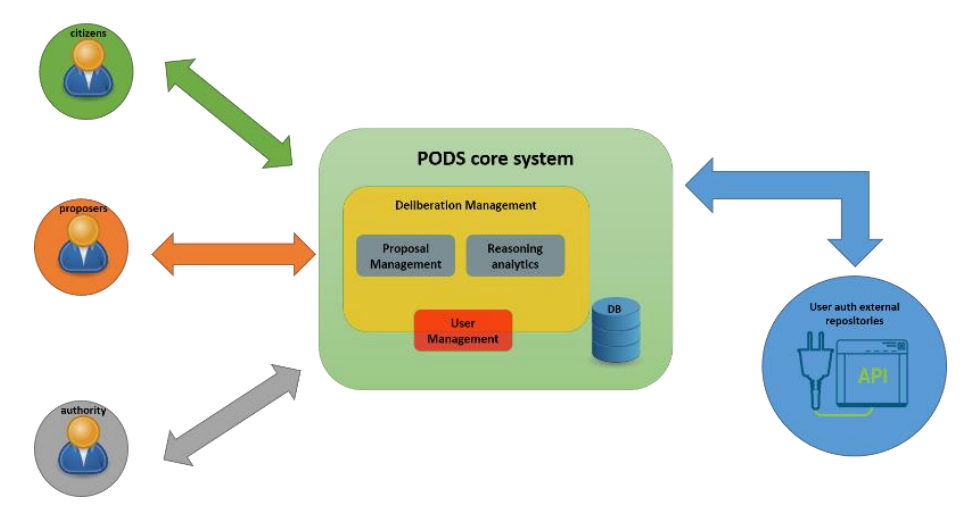

Figure 1. PODS conceptual architecture

\section{PODS EVALUATION PROCESS}

The PODS evaluation process is designed and implemented a model which is presented below. It is organized under the following schema. All phases are time constrained, meaning each phase is complete into a specified time frame, different for each theme deliberation.

1. Initialization. The system administrator initiates the system by providing relevant information about the participants (name, location information, expertise, relevance, participation frequency, digital reputation etc.). The above information is used by the system to ensure access to eligible participants (citizens of a specific local community, members of a social group etc.) and by the authorities that have ordered the deliberation which need to setup the parameters and factors that will be used throughout the evaluation process to point out the "winning proposals".

2. Theme post. The deliberation authority sets up a concept / problem / issue that is of interest to the local government. Any registered user can introduce a theme into the PODS system. The authority can issue from time to time calls inviting any interested individual to propose a theme for discussion. The theme is included in a list after approval of the deliberation authority. A list of active themes is always available to the users. The list includes the theme title, any documentation provided by the deliberation authority, the stage it is in (proposals, evaluation of proposals, merging etc.) and the time remaining to complete the specific stage.

3. Proposal post. In this phase every participant can participate by introducing for each theme a proposal. All proposals with relevant documentation are displayed under a proposal list that is attached to each theme. For a large scale deliberation scenario (e.g. a theme that concerns a country), the deliberation authority can choose to pose a pre-theme post phase. During this phase, the writers of the proposals shape their suggestion and invite other users to support it. A proposal passes to the next phase only if it gathers an adequate number of supporters (the exact threshold is defined during the initialization phase by the deliberation authority). Following this workflow, only mature-enough proposals enter the evaluation phase.

4. Evaluation phase. This phase is the core of the system. It includes three major steps:

i. Vote on a proposal. Each participant has the opportunity to perform a vote on a proposal. Although in certain electoral systems participants may be allowed to cast multiple votes a citizen / participant can cast only a single vote. The total number of votes corresponds to the number of citizens who evaluate a given proposal. To any given proposal a positive, negative or neutral votes respectively. Each proposal can be evaluated based on the measure of acceptance where proposals having sufficient positive attraction are considered as candidates to qualify to the next phase.

Participants can add comments or add relevant documentation supporting their vote or suggest proposal merging. Proposal merging is a process where proposals that seem similar in context are indicated by participants and the relevant proposers are in order to encourage them to present a new proposal as a result of the comments of the participants. Proposers are notified if their proposal is recommended for "merging". 
The whole voting process follow the basic principles applied by all voting systems (Bouras, Katris, \& Triantafillou, 2003), (Cranor \& Cytron, 1997), (Fujioka, Okamoto, \& Ohta, 1992) and include: Accuracy, Democracy, Privacy, Verifiability, Convenience, Flexibility, Mobility and Cost Efficiency.

Accuracy: A voting system is considered accurate when (1) no one can alter a casted vote since it has been submitted to the system, (2) a valid vote cannot be miscounted, deleted or removed from the final tally and (3) an invalided vote form cannot be counted in the tallying procedure.

Democracy: A voting scheme is democratic when (1) the right to cast a vote in the system applies only to legit voters and (2) the system ensures that each legit voter has voted once.

Privacy: A system that supports voting procedures is considered private when (1) no one among the election authority can link a vote to the individual that cast it and (2) no one of the voters is able to prove that an individual voted in a certain way.

Verifiability: A system is verifiable since all voters independently can verify that all votes have been counted correctly during the tallying process.

Convenience: A voting system is considered convenient if (1) it is easy to use and (2) it does not require from the potential users to have explicit hardware.

Flexibility: A system is flexible when it allows a number of voting procedures.

ii. Application of political analytics to be used for evaluating the proposal submitted either by the local government or by citizens. The general principles behind any realistic implementation of political analytics are presented in (Drakopoulos, Triantafyllou, \& Christodoulos, 2018), (Easton), (Moscrop \& Warren, 2016) and are set either by the participants or the local government (based on political decisions). The decision criteria and factors should be transparent to users in order to reinforce a culture of openness and accountability.

iii. After the rating and acceptability phases the remaining proposals are inserted back in the evaluation phase until a winning proposal pops up and is publicized.

5. Rating phase: The proposals are rated. The system displays at least the number of votes per proposal, the criteria and factors used to rank proposals and the way the proposals were merged or rejected. The results are presented categorized by various criteria. This is a fundamental requirement since the analysis of the votes depends on a number of socioeconomic criteria beyond the age, gender, and income. Today factors such as education, work experience, religious views, political alignment, exposure to social media, and family size play an important yet unconscious role in daily decision making. This allows the local government authorities to analyze the results at this phase. Also, at this phase, intelligent modules perform multivariate analysis of the collected data, which aims to reveal new knowledge about the citizens' opinions and trends.

6. Acceptability phase. This a repetitive phase which pops up the "winning proposals". At each round the last ranked proposal is deleted and a new round begins with the remaining proposals graded by the participants and ranked according to their acceptance between individuals. The cycle ends when a "winning proposal" surfaces having the majority of acceptance between individuals.

7. Publication phase. At this phase the outvoted proposal is presented. The proposal is accompanied by documented information that can be used by the local government in supporting the relevant proposal. The analysis of the votes depends on a number of socioeconomic criteria beyond the age, gender, and income. Today factors such as education, work experience, religious views, political alignment, exposure to social media, and family size play an important yet unconscious role in daily decision making.

The whole process is based on a repetitive schema which is composed of consecutive rounds. Each round after evaluating and grading results in a set of acceptable proposals. The last proposal graded is deleted and a new round is introduced. The new round includes remaining proposals (undeleted) of new proposals (resulting from the merging process). After several rounds a final outvoted proposal surfaces and it is presented. The final proposal is presented accompanied with all relevant documentation to the authority and the decision makers. Decision makers have access to the whole process data which can be used for further review. Figure 2 represents the process workflow which denotes the interaction between users and processes. 


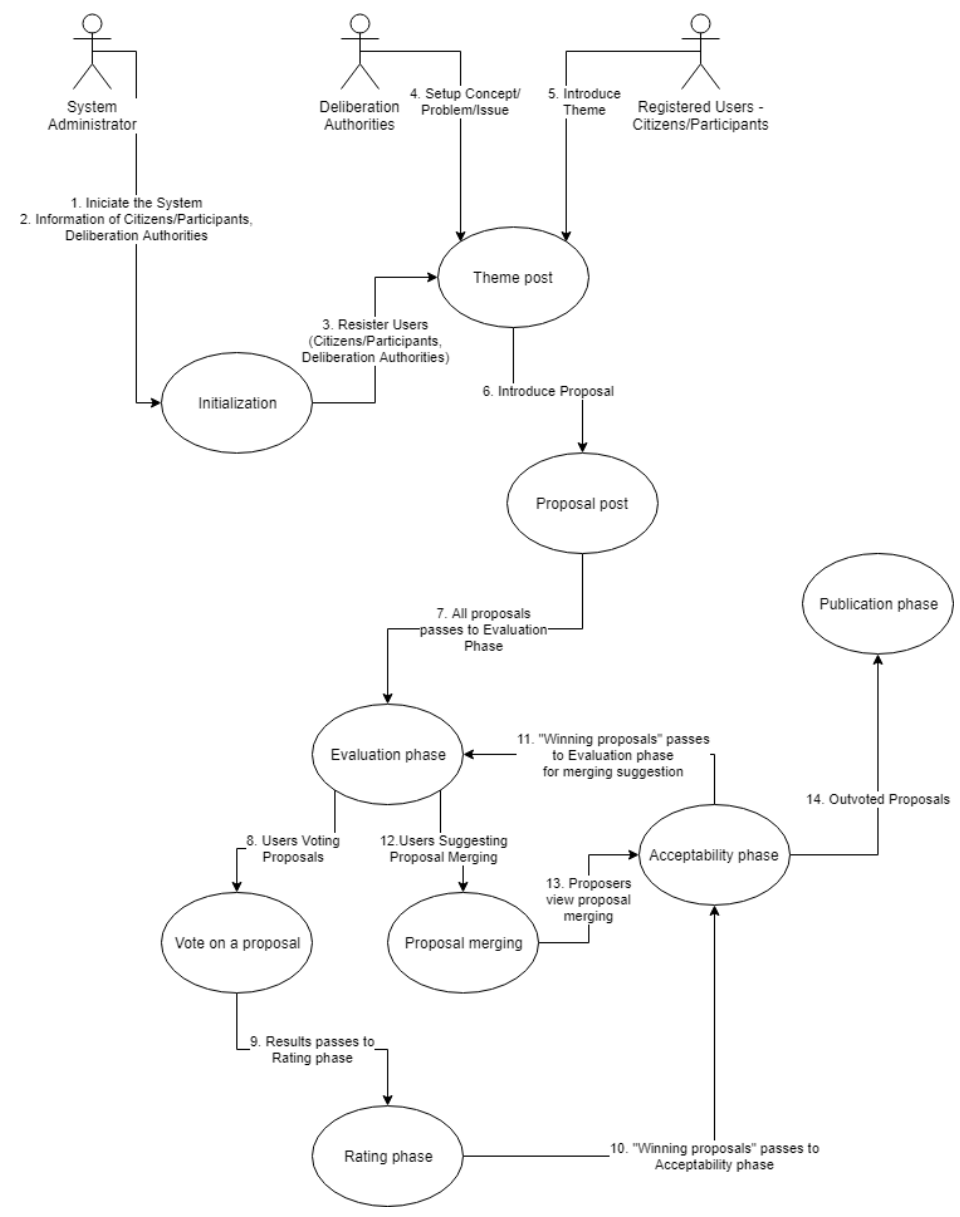

Figure 2. Process workflow

\section{CONCLUSIONS AND FUTURE WORK}

It can be easily stated that e-deliberation is the key procedure / process to broaden people's awareness and without doubt fundamental for our democracy. Nevertheless, the whole procedure ought to ensure a number of crucial criteria such as integrity, visibility and transparency. PODS schema provides a robust way of applying e-deliberation for local governments.

We intend to implement and test the PODS system at a municipal level applied in real problems. Our research will extend to how the system will incorporate a series of fundamental and advanced political analytics which are based on significant social factors. These factors will be researched in order to define criteria and factors that influence the selection process. Specifically, the roles of positive and neutral votes, geolocation information, citizen proposed keywords and comments, participation frequency, digital reputation and other factors are to be explored. Research also will focus on how to design and develop a more generic PODS system in order to support deliberation in different concepts and setups, like local government (solution vs. political cost), organizations, enterprises (e.g. strategic decisions), public groups (for evaluation prioritization of subjects to attack), entertainment section (proposals for travel, restaurants, etc.) and marketing campaigns (public acceptance). 


\section{ACKNOWLEDGEMENT}

This research is supported by "An Effective Public Deliberation System", a project funded under Research and Innovation Strategies for Smart Specialisation (RIS3) act, region of Western Greece.

\section{REFERENCES}

Bouras, C., Katris, N., \& Triantafillou, V. (2003). An e- voting service to support decision-making in local government. Telematics and Informatics, 20, 255-274.

Council, N. C. (2008, 06). Deliberative public engagement: nine principles. Retrieved 02 01, 2019, from Involve: https://www.involve.org.uk/sites/default/files/field/attachemnt/Deliberative-public-engagement-nine-principles.pdf

Cranor, F. L., \& Cytron, K. R. (1997). Sensus: A security -conscious Electronic polling system for the Internet. Hawaii I International Conference on System Science. Hawaii.

Drakopoulos, G., Triantafyllou, V., \& Christodoulos, J. (2018). Political analytics for public electronic proposal deliberation at municipal level. IADIS International Conference ICT, Society, and Human Beings. Madrid.

Easton, D. (n.d.). An approach to the analysis of political systems. World Politics, 9(3), 383-400.

Fujioka, A., Okamoto, T., \& Ohta, K. (1992). A practical secret voting scheme for large scale elections. In J. Seberry, \& Y. Zheng (Eds.), Advances in Cryptology (pp. 244-251).

Institute, C. (2010). The Consultation Charter. In C. Institute.

Macintosh, A. (2004). Characterizing E-Participation in Policy-Making . Proceedings of the 37th Hawaii International Conference on System Sciences.

Macintosh, A. (2006). eParticipation in policy-making: the research and the challenges. Exploit. Knowl. Econ. Issues, Appl. Case Stud., 364-369.

Moscrop, D., \& Warren, M. (2016). When is deliberation democratic. Journal of Public Deliberation, 12(2).

Poland, P. (2001). Online Consultation In GOL-IN Countries - Initiatives to foster e-democracy. Amsterdam: The Netherlands: Ministry of the Interior and Kingdom Relations.

Verdiesen, I. C. (2016). MOODs: Massive Open Online Deliberation - A Practical Application. EDIA@ECAI. doi:10.13140/RG.2.1.3030.8085 